\title{
G

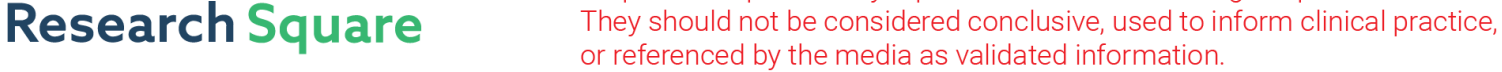 \\ Prediction of death after multiple wasp stings with a clinical prognostic model
}

\section{Maohe Wang}

Suining Central Hospital

\section{Mei Qin}

Suining Central Hospital

\section{Amanda Y Wang}

Renal and Metabolic Division, The George Institute for Global Health, UNSW

\section{Jia Wei Zhao}

The faculty of Health Sciences and Medicine, Bond University, QLD

\section{Fei Deng}

Renal Department and Nephrology Institute, Sichuan Provincial People's Hospital, School of Medicine, University of Electronic Science and Technology of China, Chengdu

\section{Guisen Li}

Renal Department and Nephrology Institute, Sichuan Provincial People's Hospital, School of Medicine, University of Electronic Science and Technology of China, Chengdu

\section{Wei Wang ( $\nabla$ wangweisz@med.uestc.edu.cn)}

Renal Department and Nephrology Institute, Sichuan Provincial People's Hospital, School of Medicine, University of Electronic Science and Technology of China, Chengdu

\section{Research Article}

Keywords: wasp sting, poisoning severity score, death, prognostic, nomogram

Posted Date: October 19th, 2021

DOI: https://doi.org/10.21203/rs.3.rs-877961/v2

License: (c) (i) This work is licensed under a Creative Commons Attribution 4.0 International License. Read Full License 


\section{Abstract \\ Background}

We aimed to assess the utility of the poisoning severity score (PSS) as early prognostic predictors in patients with wasp stings, and to explore a reliable and simple predictive tool for short-term outcomes.

\section{Methods}

From January 2016 to December 2018, 363 patients with wasp stings in Suining Central Hospital were taken as research subjects. In the first $24 \mathrm{~h}$ of hospital admission, the PSS and Chinese expert consensus on standardized diagnosis and treatment of wasp stings (CECC) were used as the criterion for severity classification, and their correlation was analyzed. The patients were divided into survival and death groups according to the state of discharge. The factors that affect outcome were analyzed by logistic regression analysis. A clinical prognostic model of death was constructed according to the risk factors, and 1000 times repeated sampling was done to include the data to verify the model internally.

\section{Results}

The mortality of wasp sting patients was 3.9\%. There was a correlation between PSS and CECC $(r=0.435$, $P<0.001)$ for severity classification. Sex, age, number of stings, and PSS were independent risk factors for death. Based on the 4 independent risk factors screened by the above regression analysis, a nomogram model was constructed to predict the risk of death in wasp sting patients. The predicted value C-index was 0.962 , and the internally verified AUC was $0.962(95 \%$ C.I. $0.936-0.988, P<0.001)$.

\section{Conclusions}

PSS is helpful in the early classification of the severity of wasp stings. Sex, age, number of stings, and PSS were independent risk factors for death in wasp sting patients. The nomogram model established in this study can accurately predict the occurrence of the risk of death.

\section{Background}

China is a large agricultural country. With developments in returning farmland to forests in rural areas, vegetation is becoming more and more abundant, providing a suitable habitat for wasps. Thus the incidence of wasp stings is increasing, bringing a serious burden to people's public health and social economy[1, 2]. Patients with wasp stings have a variety of clinical symptoms, including redness, swelling, pain, anaphylactic shock, rash, and allergies[3, 4]. The mortality after wasp stings ranged from $5.1-21 \%[1$, 5]. It is particularly important to classify the severity at an early stage and to carry out the corresponding treatment[6,7]. Severity of wasp stings and its clinical course may not be predicted at presentation. A tool 
combined with clinical and biochemical parameters may assist physicians in instituting appropriate management and predict outcomes. SOFA and APACHEII scores have been used to classify the severity of wasp sting patients $[3,4,8]$. However, as the purpose of these systems was to identify patients at risk of deterioration in ICU, they are unable to identify patients with severe wasp stings at an early stage. In the "Chinese expert consensus on standardized diagnosis and treatment of wasp stings (CECC)" published in 2018, a guide to classifying the severity of wasp stings was described, but it has not been widely used[6]. In Europe, the poisoning severity score (PSS) was used to assess the severity of poisoning patients (including environmental toxins) [9]. Stay in observation, hospitalization, admission to ICU or general ward and nursing grade were decided according to the assessment results[10]. However, to the authors' knowledge, no previous studies have specifically addressed the use of PSS for evaluating the severity of wasp sting patients. Therefore, we undertook this study in which we looked at 363 patients with wasp stings from January 2016 to December 2018 in Suining Central Hospital, to assess the utility of the PSS as early prognostic predictors of short-term outcomes. This study also explored a reliable and simple predictive tool to identify patients at a high risk of death, to optimise patient management to reduce the fatality rate.

\section{Methods}

\section{Research subjects}

This was a retrospective study of patients with wasp stings who presented to the nephrology department and ICU of Suining Central Hospital of Sichuan Province, China between January 2016 to December 2018. Suining Central Hospital of Sichuan Province, China, is the only tertiary grade A general hospital in the interior areas of Sichuan Province that has more than 94,000 annual hospitalized patients and more than 100 wasp sting patients each year.

The inclusion criteria were: 1) Patients with a definite diagnosis of wasp stings; 2 ) age $\geqq 14$ years old; 3 ) the clinical data were complete. The exclusion criteria were as follows: 1) age<14years; 2) re-hospitalized patients with wasp stings; 3 ) wasp sting patients who died before admission. We categorized them into survival group ( $n=349)$ and death group $(n=14)$ according to the state of discharge. This study was approved by the Ethics Committee of Suining Central Hospital (Serial number: LLSNCH20200022).

\section{Clinical data collection}

We collected information on the patients' demographics (age, sex), the time interval between sting and admission (admission time), number of stings, signs and symptoms (allergic rash, hypotension, macroscopic hematuria, and oliguria or anuria), severe complications (rhabdomyolysis, acute kidney injury (AKI), coagulation disorders, hemolysis, liver dysfunction, acute respiratory distress syndrome (ARDS) and multiple organ dysfunction syndrome (MODS)), inpatient days, and short-term outcomes (death or survival). 
We recorded laboratory data on admission, including: white blood cells (WBC), activated partial thromboplastin time (APTT), prothrombin time (PT), alanine aminotransferase (ALT), aspartate aminotransferase (AST), indirect bilirubin (I-BIL), creatine kinase (CK), lactate dehydrogenase (LDH), the serum creatinine ( $\mathrm{SCr}$ ).

\section{At admission, PSS and CECC were used as the criterion for severity classification respectively}

The PSS grades severity as (0) none, (1) minor, (2) moderate, (3) severe, and (4) fatal poisoning. The severity grading takes into account only the observed clinical symptoms and signs, but does not estimate risks or hazards on the basis of information such as the amount ingested or serum concentrations of the toxic agent ${ }^{[11]}$. According to the admission criteria, patients with no symptoms and those who died before admission were excluded. Thus the PSS graded the patients into minor, moderate, and severe poisoning levels.

The CECC grades severity as: (1) minor: the number of stings was less than 10, with only local allergic reactions and no organ function involvement, (2) moderate: the number of stings was between 10 and 30; allergic reaction was classified as $\mathbb{Q}-\mathbb{Q}$, only 1 organ was involved; sequential organ failure score (SOFA) $\geq 2$ points; there were macroscopic hematuria in an early stage, (3) severe: the number of stings was greater than 30; allergic reaction was classified as $₫-\mathbb{\bigotimes}$ or at least 2 organs involved; SOFA $\geq 2$ points for each organ ${ }^{[6]}$.

\section{Statistical analysis}

Continuous variables with normal distribution were expressed as means and standard deviations. Categorical variables without normal distribution were expressed as medians and interquartile ranges. Variables of the two groups were compared by Mann-Whitney $U$ test. Spearman analysis was performed for the correlation between PSS and CECC. ROC curve analysis of PSS and CECC was performed respectively, and Z-test was used to analyze the difference of AUC between them.

Univariate and multivariate logistics regression were used to analyze the risk factors of death in wasp sting patients. On the basis of the final model, a nomogram was built using $\mathrm{R}$ software with the rms package. A concordance index (C-index) was used to determine the discrimination of the nomogram, and it was calculated by a bootstrap approach with 1,000 resamples for internal validation. Receiver operating characteristic (ROC) curves were plotted, and the areas under the ROC curve were calculated.

A p-value less than 0.05 was considered statistically significant.

\section{Results}

\section{Descriptive Results}


Between January 2016 to December 2018, a total of 390 patients were identified with wasp stings. Of these patients, 27 patients were excluded, including three who died on admission and 24 who refused to be hospitalized. Finally, 363 patients were included in this study, which included $219(60.3 \%)$ males and $144(39.7 \%)$ females. The mean age was $55.9 \pm 16.3$ years. Fourteen(3.9\%) patients died during hospitalization, including 1 in (PSS) grade 1, 9 in (PSS) grade 2, and 4 in (PSS) grade 3. Over the threeyear period of this study, the deaths only occurred from September to November. (Fig. 1, Table3)

\section{PSS and CECC}

The median PSS was 1(1,1) upon admission. grade 1 (minor) PSS upon admission were seen in 290 $(79.9 \%)$ cases, grade 2 (moderate) in $59(16.2 \%)$ cases and grade 3 (severe) in 14 (3.9\%) cases. (Table1)

Regarding the CECC assessment upon admission, minor condition was found in 131 (36.1\%) cases, moderate in 176 (48.5\%) cases, severe in $56(15.4 \%)$ cases. (Table1)

\section{Spearman analysis between PSS and CECC}

The Spearman analysis showed the correlation between PSS and CECC $(r=0.435, P<0.001)$, which indicated that there was a correlation between PSS and CECC in assessing the severity of wasp sting patients. (Table1)

\section{ROC curve analysis of PSS and CECC}

The AUC of the PSS and CECC in predicting death in wasp sting patients was 0.890 and 0.845 , respectively, and they showed a certain predictive power. Although the AUC of the PSS was better than that of CECC, the difference between them was not statistically significant ( $Z=0.7230, P=0.4697)$. Table 2 showed the optimal cut off value, sensitivity, specificity, positive predictive value, and negative predictive values of PSS and CECC. (Fig. 2, Table 2).

\section{Comparison of clinical data between the survival and the death group}

The death group had a greater proportion of females ( $85.7 \%$ vs. $37.8 \%, P<0.001)$, and was significantly older $(71.1 \pm 9$.8years vs. $55.3 \pm 16.2$ years, $P<0.001)$ than that of the survival group. The number of stings (30 vs. $8, P<0.001$ ) and the time from stings to admission ( $7 \mathrm{~h}$ vs. $3 \mathrm{~h}, \mathrm{P}=0.004$ ) in the death group were higher than those in the survival group $(P<0.001)$. The PSS of the death group was significantly higher than that of the survival group ( 2 vs. $1, p<0.001)$. The length of hospital stay was significantly shorter in the death group ( 1 day vs. 4 days, $P<0.001$ ). (Table3)

\section{Comparisons of complications between the survival and the death group}

No allergic rash was seen in the death group ( $0 \%$ vs. $14.3 \%, P=0.232)$. One patient in the death group developed hypotension compared to 19 in the survival group ( $P=0.554)$. The incidences of rhabdomyolysis, hemolysis, liver dysfunction, coagulation disorder, ARDS, MODS, oliguria (or anuria) and 
macroscopic hematuria in the death group were significantly higher than those in the survival group ( $\mathrm{P}<$ 0.001). Seven (1.9\%) patients were admitted to ICU, including three in the death group and four in the survivor group. The incidence of AKI in the death group was significantly higher (78.6\%) than that in the survival group $(8 \%)(P<0.001)$. A larger proportion of patients in the death group (ten patients $(71.4 \%))$ received hemodialysis compared to that in the survival group (35 patients $(10 \%))(p<0.001)$. (Table3)

\section{Comparisons of biochemical parameters between the survival and the death group}

In the first $24 \mathrm{~h}$ of hospital admission, the laboratory parameters including WBC, APTT, PT, ALT, AST, IBIL, $\mathrm{CK}, \mathrm{LDH}, \mathrm{LDH}, \mathrm{SCr}$ values in the death group were significantly higher than those in the survival group $(p<0.05)$. (Table4)

\section{Univariate logistic regression analysis}

Based on the pre-specified hypothesis that season was one risk factor for death in wasp sting patients, as such deaths only occur in summer and autumn, there were significant statistical differences in age, sex, number of stings, admission time, PSS and biochemical parameters between the death group and the survival group. However, the evaluation criteria of PSS included cardiovascular system, respiratory system, liver, kidney, muscular system and so on. Therefore, we took the patients' age, sex, number of stings, admission time, PSS and season as independent variables to perform univariate logistic regression. The results were shown in Table 5.

\section{Multivariate logistic regression analysis}

The backward multivariate logistic regression analysis showed that female sex, age, number of stings and PSS were independent risk factors ( $\mathrm{OR}=8.651,1.103,1.033,6.768$ respectively). (Table6)

A nomogram was built using $\mathbf{R}$ software with the rms package

Based on the 4 independent risk factors screened by the above regression analysis, a nomogram model was built using $\mathrm{R}$ software with the rms package to predict the risk of death in wasp sting patients, and the risk prediction ability of the model was verified. (Fig. 3)

The predicted value C-index was 0.962 , indicating that the clinical prognostic model had a good prediction accuracy and discrimination. (Fig. 4)

The internally verified AUC was $0.962(95 \%$ C.I. $0.936-0.988, P<0.001)$, indicating that the nomogram had a high predictive value. (Fig. 5)

\section{Discussion}

In China, Nepal, Vietnam and other Asian countries, systemic organ damage or even death due to wasp venom is not rare $[1,5,12]$. This retrospective study found that the mortality rate in wasp sting patients 
was $3.9 \%$, and death was only seen in the summer and autumn months from September through November among this cohort. Of the 14 deceased patients, all died between the 1st-3rd day after admission. The PSS in the death group was significantly higher than that of the survival group. Multivariate logistic regression analysis showed that female sex, increased age, higher number of stings and greater PSS grade were independent risk factors for death in wasp sting patients.

Wasp venom contains a variety of bioactive components, such as peptides, enzymes and amines[3,13]. Severe wasp stings can lead to systemic allergic reaction, rhabdomyolysis, shock, hemolysis, acute kidney injury (AKI), and even death $[2,14,15]$. Unfortunately, there is no specific antidote for wasp venom at present. It is especially important to classify the severity of wasp sting patients at an early stage, and to carry out the corresponding treatment. Patients with severe wasp stings should be promptly referred to a medical institution that can perform blood purification treatment, which can reduce mortality $[6,16,17]$. Chinese Society of Toxicology had prepared a consensus statement on the standardized diagnosis and treatment of wasp stings (CECC) in 2018[6]. Nevertheless, a wider application of this consensus criteria is likely limited by the complex evaluation criteria. In Mong's literature, the use of poisoning severity score (PSS) for the assessment of the severity of the poisoning patients (including wasp sting patients) in the emergency department was reported[10]. In the present research, there was a correlation between PSS and CECC in assessing the severity of wasp stings. The severity of wasp stings was evaluated by PSS, including 59(16.2\%) patients with grade 2 and $14(3.9 \%)$ patients with grade 3 , where a severity poisoning $\geq$ grade 2 (PSS) might lead to death. The severity of wasp stings was evaluated by CECC, including $56(15.4 \%)$ patients with severe wasp stings, where a severity poisoning $\geq$ severe (CECC) might lead to death. PSS had higher accuracy and sensitivity value (89.0\%, 92.9\% respectively), whilst CECC had a higher specificity value (86.8\%) when predicting the short-term clinical outcome in wasp sting patients. The results revealed that these two criteria determined the severity of poisoning and were able to predict the short-term clinical outcomes due to wasp stings. As the PSS was more predictive and simpler, it is recommended to poisoning centers as effective criteria for classify the severity of wasp sting patients.

Wasp stings were the main cause of human death caused by animal injuries, and it was the main cause of community-acquired AKI in Asia[16]. In this study, the mortality of our wasp sting patients was $3.9 \%$, which was consistent with Xie, etc.'s report[1]. When the severity was graded according to the PSS, the mortality was as high as $28.5 \%$ in the severe group and $15.2 \%$ in the moderate group. PSS was an independent risk factor for death in wasp sting patients. The severity was also related to the number of stings. In Xie and Liu, etc.'s literature, the overall incidence of severe complications was higher in the group with more than 10 stings $[1,18]$. In our patients, the mean number of stings in the death group was 30 . Females accounted for $70 \%$ of patient deaths. Multivariate logistics regression showed that the number of stings and female sex were also independent risk factors for death in wasp sting patients. The average age in the death group was significantly higher than that in the survival group. As such, age was also one of the risk factors for death, which may be related to underlying diseases in elderly patients. The four indexes of female sex, age, the number of stings, and PSS were combined as prediction criteria, and predicting the death of wasp sting patients had high accuracy (AUC=0.962, 95\%C.I. 0.936-0.988, $\mathrm{P}<0.001)$, which was more powerful than using PSS (AUC=0.890) or CECC $(A U C=0.845)$ alone. Therefore, 
a nomogram prediction model was built on the base of independent risk factors determined through logistic analyses. The predicted and observed values were found to be similar, which indicates that this prediction model demonstrates a good degree of discrimination and calibration. Clinicians can identify the patients at high risk of death for patient management and reduce the fatality rate according to this nomogram. It is recommended to poisoning centers as effective criteria.

\section{Limitations Of This Study}

Our study has some limitations. First, as this was a single-center retrospective study, there may be selection bias in addition to possible confounding. Second, our prediction model only takes into account the relevant clinical data on admission, and does not consider the impact of previous diseases and treatment on the prognosis. Multi-center prospective studies will be needed to verify the accuracy of this model in future research.

\section{Conclusions}

This retrospective cohort study demonstrated that PSS is helpful in early classification the severity of wasp sting patients. Female sex, age, number of stings, and PSS were independent risk factors for the death of wasp stings. The nomogram model established in this study can accurately predict the occurrence risk of death.

\section{Abbreviations}

PSS

poisoning severity score

CECC

Chinese expert consensus on standardized diagnosis and treatment of wasp stings AKI

acute kidney injury

ARDS

acute respiratory distress syndrome

MODS

multiple organ dysfunction syndrome

WBC

white blood cells

APTT

activated partial thromboplastin time

PT

prothrombin time (),ALT:alanine aminotransferase

AST

aspartate aminotransferase 
I-BIL

indirect bilirubin

CK

creatine kinase

$\mathrm{LDH}$

lactate dehydrogenase

$\mathrm{SCr}$

the serum creatinine.

\section{Declarations}

\section{Ethics approval and consent to participate}

The study was approved by the Ethics Committee of Suining Central Hospital (Suining, China). and all the patients signed informed consents to participate in this study. (LLSNCH20200022)

\section{Consent for publication}

Not applicable.

\section{Availability of data and material}

All data and material were obtained from Suining Central Hospital.

\section{Competing interests}

The authors report no conflicts of interest.

\section{Funding}

This work was supported by a grant from the University of Electronic Science and Technology of China Central University Research Fund (ZYGX2019J104), the Science and Technology Project of Sichuan Province (2020YJ0447), the National Natural Science Foundation of China (No. 81970641), Sichuan Medical Research Project(S18040), the Science and Technology project of the heath planning Committee( 19PJ132) and the Renal Department and Institute of Nephrology, Sichuan Provincial People's Hospital, University of Electronic Science and Technology of China, Sichuan Clinical Research Center for Kidney Diseases(2019YFS0538). Dr Amanda Y Wang is supported by the National Heart Foundation PostDoctoral Fellowship and RACP jacquot Research Establishment Fellowship Australia.

\section{Authors' contributions}

Data collection (MHW, MQ, FD, WW), study design (WW, GSL,FD), statistical analyses (MHW, WW), writing (MHW囚AYW, JWZ,WW) Ølanguage modification (AYW, JWZ). All authors have read and approved the manuscript. 
Acknowledgments

We are grateful to all the subjects who participated in this work.

\section{References}

1. Xie C, Xu S, Ding F, Xie M, Lv J, Yao J, Pan D, Sun Q, Liu C, Chen T et al: Clinical features of severe wasp sting patients with dominantly toxic reaction: analysis of 1091 cases. PLOS One 2013, 8(12):e83164.

2. Vikrant S, Parashar A: Wasp venom-induced acute kidney injury: a serious health hazard. Kidney Int 2017, 92(5):1288.

3. Gong J, Yuan H, Gao Z, Hu F: Wasp venom and acute kidney injury: The mechanisms and therapeutic role of renal replacement therapy. Toxicon 2019, 163:1-7.

4. Zhang $L$, Yang $Y$, Tang $Y$, Zhao $Y$, Cao Y, Su B, Fu P: Recovery from AKI following multiple wasp stings: a case series. Clin J Am Soc Nephrol 2013, 8(11):1850-1856.

5. Sigdel MR, Raut KB: Wasp bite in a referral hospital in Nepal. J Nepal Health Res Counc 2013, 11(25):244-250.

6. Chinese Society Of Toxicology P, Treatment Of Specialized C, Hubei Emergency Medicine Committee Of Chinese Medical A, Hubei Provincial P, Occupational Disease U, Yang X, Xiao M: Expert consensus statement on standardized diagnosis and treatment of wasp sting in China. Zhonghua Wei Zhong Bing Ji Jiu Yi Xue 2018, 30(9):819-823.

7. Wijerathne BT, Rathnayake GK, Agampodi SB: Hornet stings presenting to a primary care hospital in Anuradhapura District, Sri Lanka. Wilderness Environ Med 2014, 25(1):122-126.

8. Yuan H, Chen S, Hu F, Zhang Q: Efficacy of Two Combinations of Blood Purification Techniques for the Treatment of Multiple Organ Failure Induced by Wasp Stings. Blood Purif 2016, 42(1):49-55.

9. Persson HE, Sjoberg GK, Haines JA, Pronczuk de Garbino J: Poisoning severity score. Grading of acute poisoning. J Toxicol Clin Toxicol 1998, 36(3):205-213.

10. Mong R, Arciaga GJ, Tan HH: Use of a 23-hour emergency department observation unit for the management of patients with toxic exposures. Emerg Med J 2017, 34(11):755-760.

11. Casey PB, Dexter EM, Michell J, Vale JA: The prospective value of the IPCS/EC/EAPCCT poisoning severity score in cases of poisoning. J Toxicol Clin Toxicol 1998, 36(3):215-217.

12. Xuan BH, Mai HL, Thi TX, Thi MT, Nguyen HN, Rabenou RA: Swarming hornet attacks: shock and acute kidney injury-a large case series from Vietnam. Nephrol Dial Transplant 2010, 25(4):1146-1150. 
13. Habermann E: Bee and wasp venoms. Science 1972, 177(4046):314-322.

14. Warrell DA: Venomous Bites, Stings, and Poisoning: An Update. Infect Dis Clin North Am 2019, 33(1):17-38.

15. Forrester JD, Forrester JA, Tennakoon L, Staudenmayer K: Mortality, hospital admission, and healthcare cost due to injury from venomous and non-venomous animal encounters in the USA: 5-year analysis of the National Emergency Department Sample. Trauma Surg Acute Care Open 2018, 3(1):e000250.

16. Yang L: Acute Kidney Injury in Asia. Kidney Dis (Basel) 2016, 2(3):95-102.

17. Zuk A, Bonventre JV: Acute Kidney Injury. Annu Rev Med 2016, 67:293-307.

18. Liu Z, Li XD, Guo BH, Li Y, Zhao M, Shen HY, Zhai Y, Wang XL, Liu T: Acute interstitial nephritis, toxic hepatitis and toxic myocarditis following multiple Asian giant hornet stings in Shaanxi Province, China. Environ Health Prev Med 2016, 21(4):231-236.

\section{Tables}

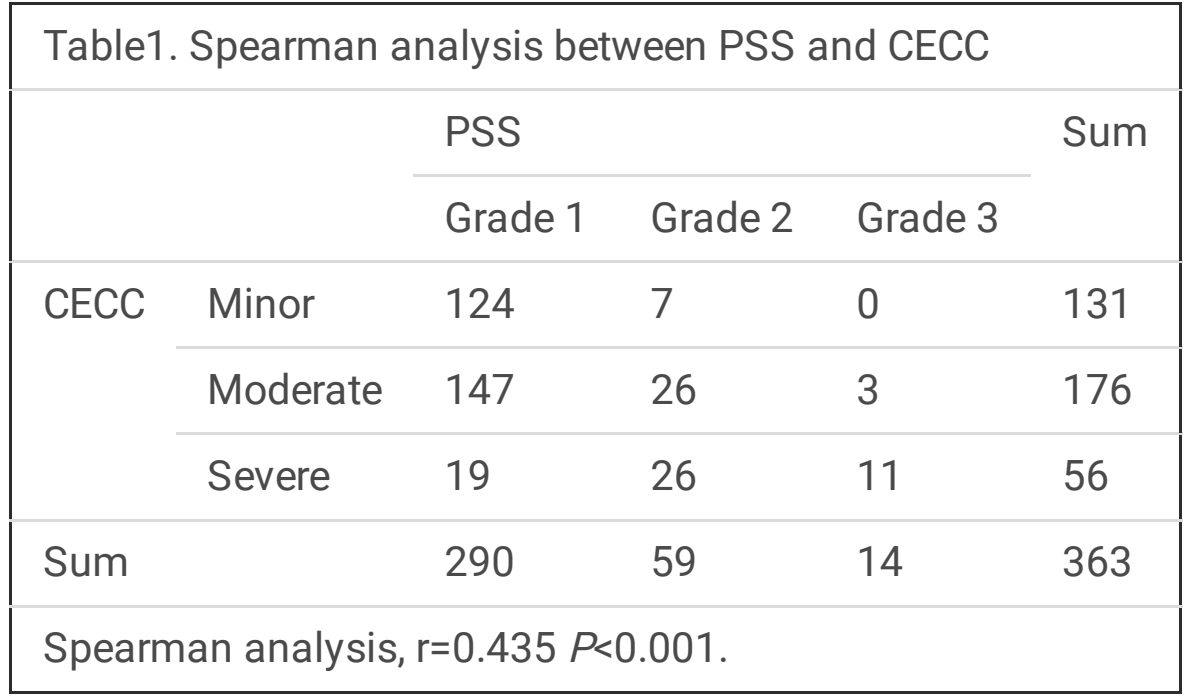

Table2. The ROC analysis of PSS and CECC in the optimal cut off scores

\begin{tabular}{|c|c|c|c|c|c|c|c|}
\hline \multirow[t]{2}{*}{ Score } & \multicolumn{5}{|l|}{ Value } & \multicolumn{2}{|c|}{ The comparison of $A U C$} \\
\hline & Sensitivity (\%) & Specificity (\%) & +PV (\%) & $-P V(\%)$ & AUC & Z & $P$ \\
\hline PSS>1 & 92.86 & 82.81 & 17.8 & 99.7 & 0.890 & 0.7230 & 0.4697 \\
\hline $\mathrm{CECC}>2$ & 71.43 & 86.82 & 17.9 & 98.7 & 0.845 & & \\
\hline
\end{tabular}




\begin{tabular}{|c|c|c|c|}
\hline Variable & Survival group $(\mathrm{n}=349)$ & Death group $(n=14)$ & $P$ \\
\hline Age (years) & $55.3 \pm 16.2$ & $71.1 \pm 9.8$ & $<0.001$ \\
\hline Gender (M: F) & $217: 132$ & $2: 12$ & $<0.001$ \\
\hline Number of stings & $8(4,15)$ & $30(20,45)$ & $<0.001$ \\
\hline Admission time (h) & $3(2,5)$ & $7(3.7,10)$ & 0.004 \\
\hline Poisoning severity score & $1(1,1)$ & $2(2,3)$ & $<0.001$ \\
\hline Grade 1 (n) & 289 & 1 & \\
\hline Grade 2 (n) & 50 & 9 & \\
\hline Grade $3(n)$ & 10 & 4 & \\
\hline Inpatient days (day) & $4(3,7)$ & $1(1,2.2)$ & $<0.001$ \\
\hline Allergic rash n (\%) & $50(14.3)$ & $0(0)$ & 0.232 \\
\hline Hypotension n (\%) & $19(5.4)$ & $1(7.1)$ & 0.554 \\
\hline $\mathrm{AKI}^{*} \mathrm{n}(\%)$ & $28(8 \%)$ & 11(78.6) & $<0.001$ \\
\hline Rhabdomyolysis n (\%) & $92(26.4)$ & $13(92.9)$ & $<0.001$ \\
\hline Hemolysis n (\%) & $49(14)$ & 12(85.7) & $<0.001$ \\
\hline Oliguria or anuria n (\%) & $18(5.2)$ & $9(64.3)$ & $<0.001$ \\
\hline Macroscopic hematuria n (\%) & $38(10.9)$ & 13(92.9) & $<0.001$ \\
\hline Coagulation abnormalities n (\%) & $126(36.1)$ & 13(92.9) & $<0.001$ \\
\hline Liver damage n (\%) & 69(19.8) & 13(92.9) & $<0.001$ \\
\hline Dialysis n (\%) & $35(10)$ & 10(71.4) & $<0.001$ \\
\hline MODS** n (\%) & $43(12.3)$ & 13(92.9) & $<0.001$ \\
\hline ARDS\# n (\%) & $4(1.1)$ & $9(64.3)$ & $<0.001$ \\
\hline ICU $\# \#$ n (\%) & $4(1.1)$ & $3(21.4)$ & $<0.001$ \\
\hline
\end{tabular}




\begin{tabular}{|c|c|c|c|}
\hline Biochemical parameters & Survival group $(n=349)$ & Death group $(n=14)$ & $\mathrm{P}$ \\
\hline $\operatorname{SCr}^{*}(59-104 \mathrm{umol} / \mathrm{I})$ & $66(55,77)$ & $78(68.5,140)$ & 0.004 \\
\hline $\mathrm{CK}^{* \star} \rrbracket 40-200 \mathrm{U} / \mathrm{I} \mathbb{\square}$ & $199(117.5,415.5)$ & $2321(407.7,5342.5)$ & $<0.001$ \\
\hline$A S T^{\star \star \star} \rrbracket 13-35 U / \mathbb{I}$ & $35.5(26,55.2)$ & $557(255,1505.5)$ & $<0.001$ \\
\hline $\mathrm{IBIL}^{\star \star \star \star}(0-18 \mathrm{umol} / \mathrm{I} \mathbb{\mathrm { V }}$ & $8.1(5.2,16.8)$ & $39.4(19.8,83.4)$ & $<0.001$ \\
\hline $\mathrm{ALT}^{\#} \llbracket 7-40 \mathrm{U} / \mathrm{I}$ & $22(17,37)$ & 286(58.5,1369.5) & $<0.001$ \\
\hline$P T^{\# \#} \otimes 11-14.5 \mathrm{~s} \rrbracket$ & $13.7(13,14.6)$ & $15(14,17)$ & 0.002 \\
\hline APTT ${ }^{\# \# \#} \rrbracket 26-40 s \rrbracket$ & $51.3(36.8,91.2)$ & $120.5(94.1,180)$ & $<0.001$ \\
\hline LDH (120-250U/I) & $215(179,277.5)$ & 1671(1195.5,2795) & $<0.001$ \\
\hline WBC\#\#\#\# (3.5-9.5*109/I) & $11.8(8.4,15.8)$ & $26.3(21.2,33.6)$ & $<0.001$ \\
\hline \multicolumn{4}{|c|}{ 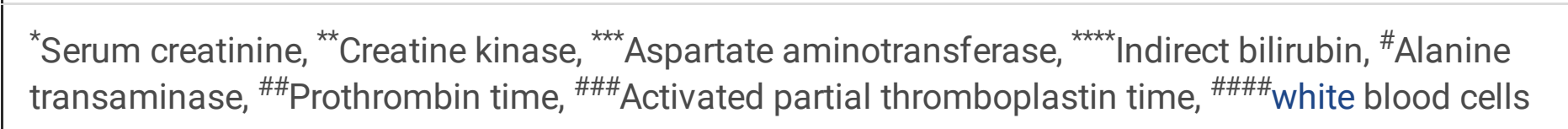 } \\
\hline
\end{tabular}

\begin{tabular}{|llllll|}
\hline \multicolumn{5}{|l}{ Table5. Univariate logistic regression analysis of risk factors of death } \\
\hline Variable & $\beta$ & Wald $\chi^{2}$ & $P$ & OR & $95 \%$ C.I. \\
\hline Age & 0.102 & 12.099 & 0.001 & 1.108 & $1.046-1.173$ \\
\hline Female & 2.289 & 8.797 & 0.003 & 9.864 & $2.174-44.762$ \\
\hline Admission time & 0.004 & 0.071 & 0.790 & 1.004 & $0.974-1.035$ \\
\hline Season & 1.694 & 5.759 & 0.016 & 5.440 & $1.364-21.699$ \\
\hline Number of stings & 0.043 & 14.808 & $<0.001$ & 1.044 & $1.022-1.068$ \\
\hline PSS & 2.135 & 29.403 & $<0.001$ & 8.460 & $3.910-18.305$ \\
\hline * & & & & & \\
\hline
\end{tabular}




\begin{tabular}{|llllll|}
\hline \multicolumn{5}{|l|}{ Table6. Multivariate logistic regression analysis of risk factors of death } \\
\hline Variable & $\beta$ & Wald $\chi 2$ & $\mathrm{P}$ & OR & $95 \%$ C.I. \\
\hline Age & 0.098 & 5.029 & 0.025 & 1.103 & $1.012-1.201$ \\
\hline Female & 2.158 & 4.885 & 0.027 & 8.651 & $1.277-58.629$ \\
\hline Number of stings & 0.033 & 4.852 & 0.028 & 1.033 & $1.004-1.064$ \\
\hline PSS & 1.912 & 9.306 & 0.002 & 6.768 & $1.981-23.120$ \\
\hline Hosmer-Lemeshow analysis: $\chi 2=0.826$ & P=0.999, ${ }^{*}$ Poisoning severity score \\
\hline
\end{tabular}

\section{Figures}

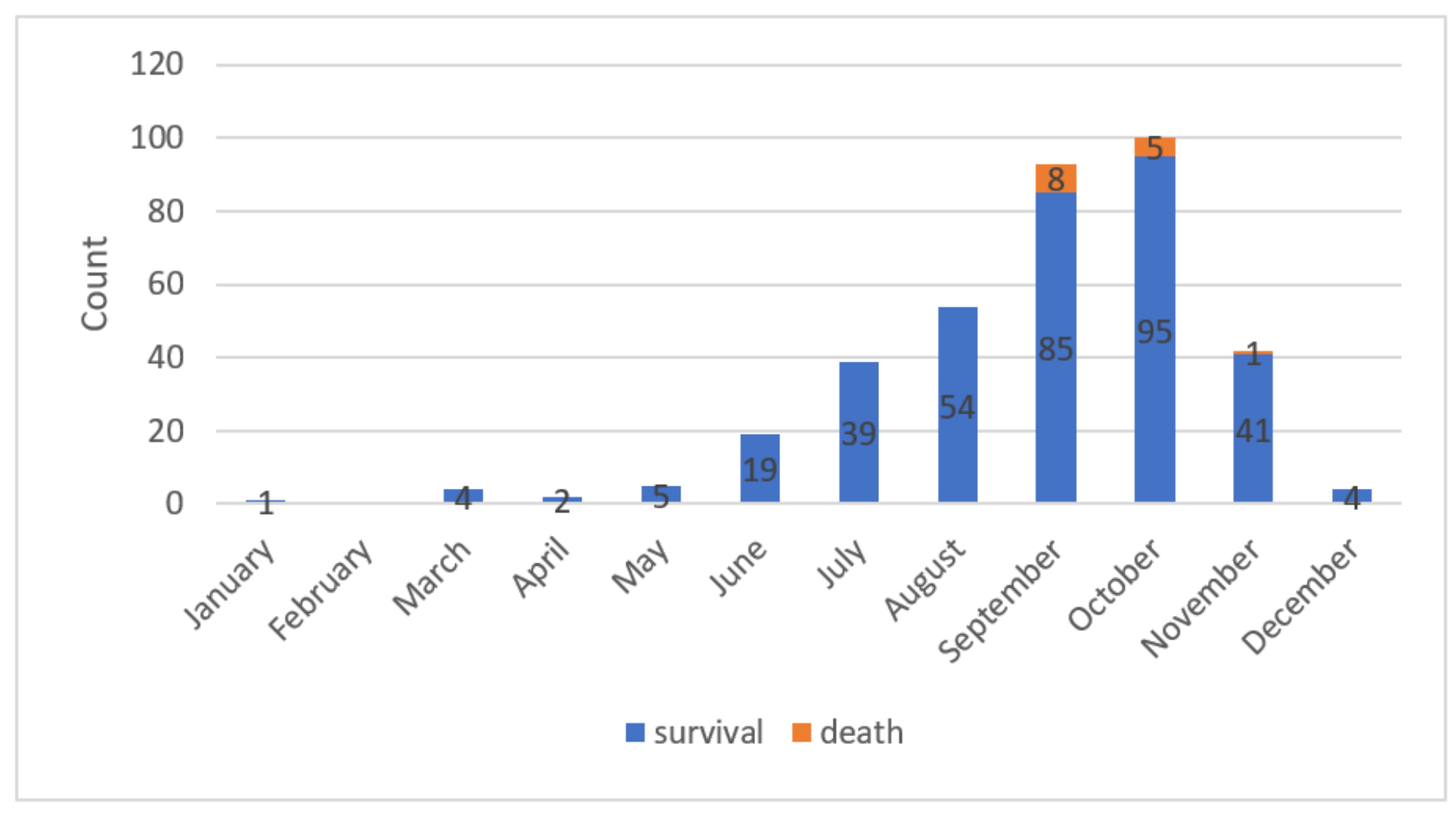

Figure 1

Monthly distribution of wasp sting patients 


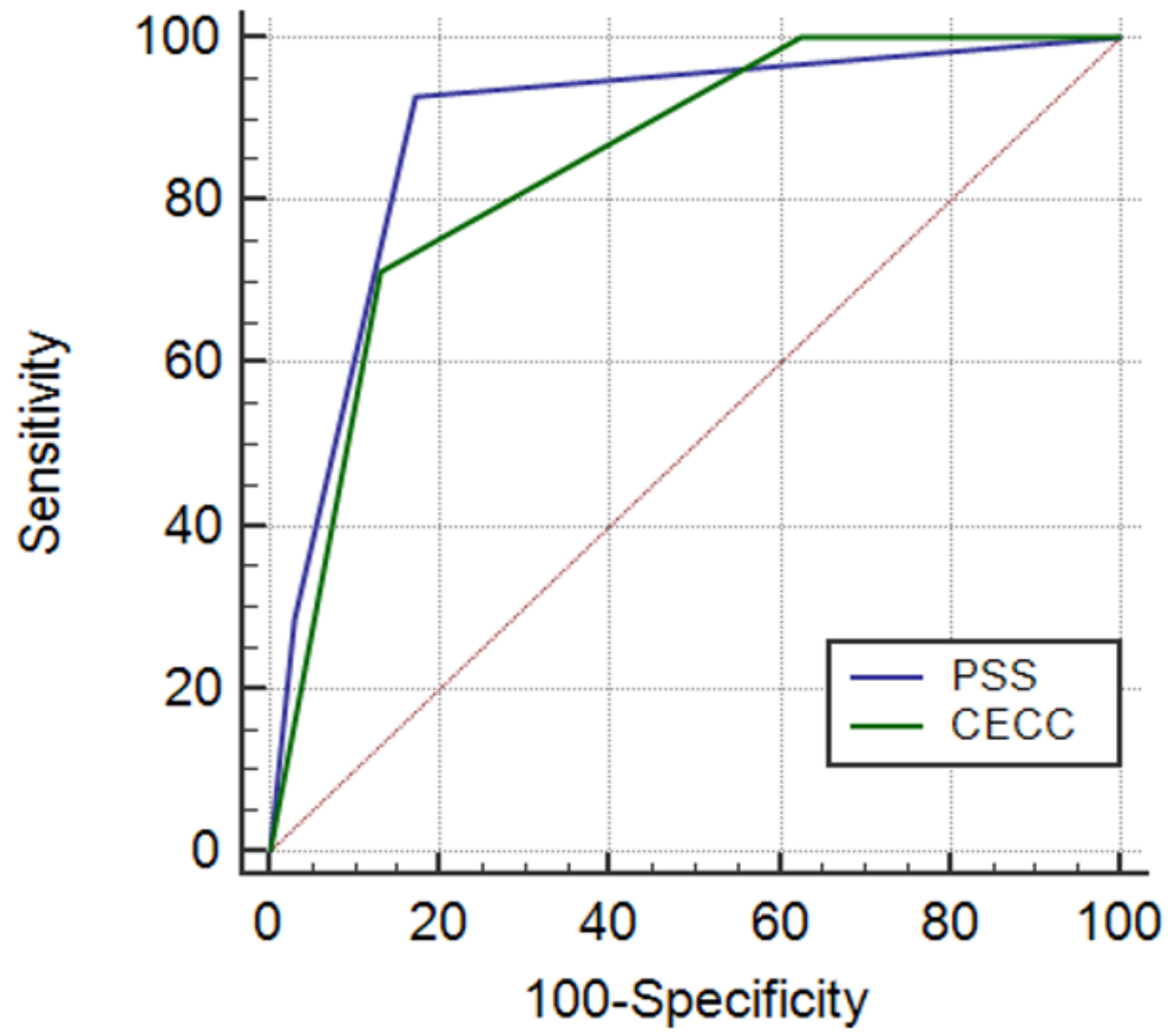

Figure 2

ROC curve analysis of PSS and CECC in predicting death 
Points

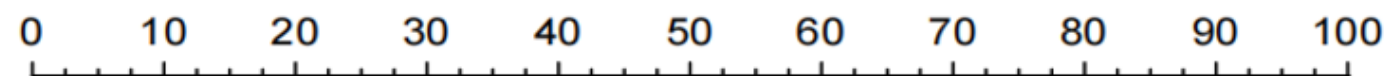

Age

Gender

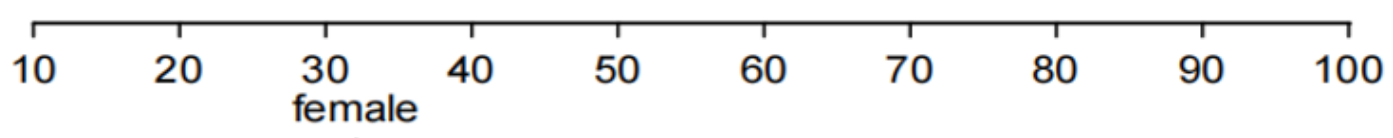

PSS

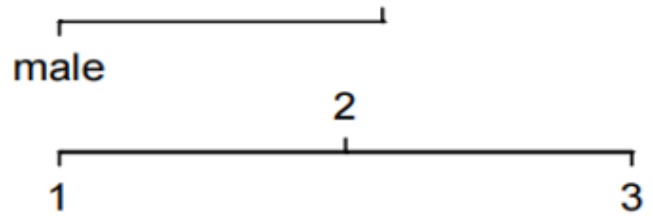

No of sting

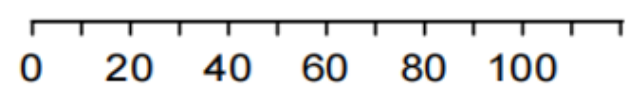

Total Points

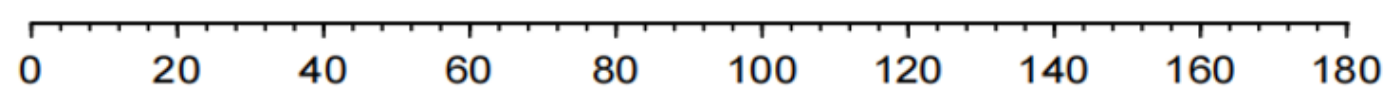

Risk

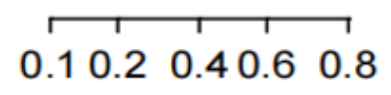

Figure 3

The nomogram model for predicting the death risk 


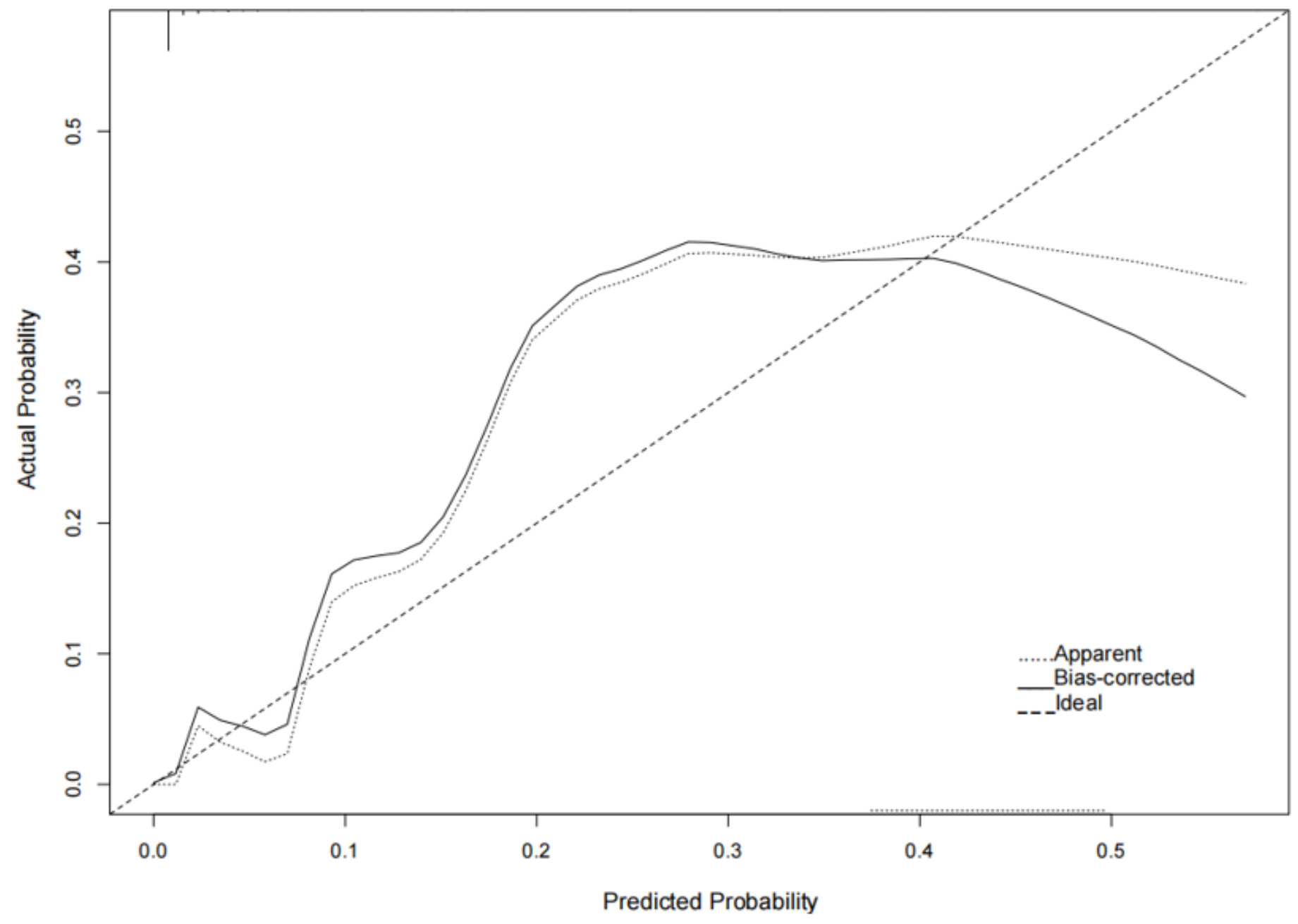

Figure 4

Verification of the nomogram model 


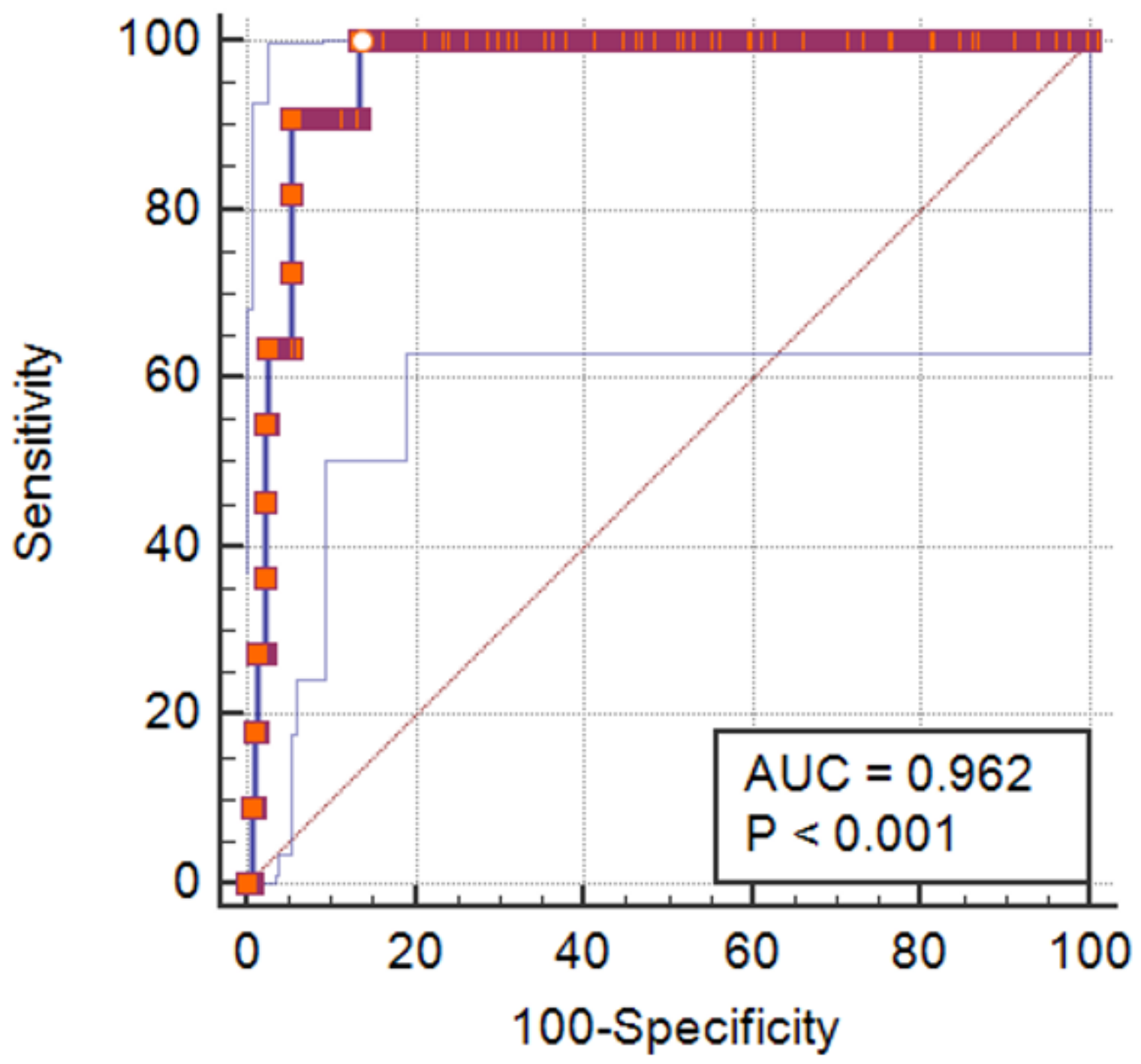

Figure 5

ROC curve analysis of the nomogram model 